\title{
Numerical and asymptotic flow stability analysis of vortex structures
}

\author{
Vadim Akhmetov
}

Moscow State University of Civil Engineering, Yaroslavskoe shosse, 26, Moscow, 129337, Russia

\begin{abstract}
Stability problem of an axisymmetric swirling flow of a viscous incompressible fluid with respect to nonaxisymmetric perturbations is considered. The system of ordinary differential equations for the amplitude functions is solved numerically by the Runge-Kutta method and orthogonalization procedure. Solutions of equations for perturbations at the neighborhood of singular points are obtained by the Frobenius method. The maximum of amplification coefficients and phase velocities of five unstable modes are calculated.
\end{abstract}

\section{Introduction}

Vortex flows are often observed in hydraulic engineering problems. The properties of the swirling flow are used in the suction tubes of hydraulic turbines, vortex spillways, countervortex energy absorbers, counter-vortex aerators, heat exchangers, purification structures, temperature and fractional separation devices [1-7]. Flow stability analysis for the considered constructions is very important and actual problem. An effective tool for studying the hydrodynamic stability of a viscous incompressible swirling flows is a model based on the Navier-Stokes equations. The hydrodynamic stability problem of swirling flows for various configurations was studied numerically in [8-15]. The results of experiments on the stability of swirling flows are presented in [16-18]. In this paper, an effective method for calculating the stability of a swirling flow with an arbitrary initial velocity profile is presented.

\section{Problem formulation}

Let's consider an axisymmetric viscous incompressible flow in a cylindrical coordinate system $(r, \varphi, z)$. The system of the Navier-Stokes equations for the variables velocity and pressure is written in the form:

$$
\begin{gathered}
\frac{\partial V_{z}}{\partial t}+V_{z} \frac{\partial V_{z}}{\partial z}+V_{r} \frac{\partial V_{z}}{\partial r}=-\frac{\partial p}{\partial z}+\frac{1}{\operatorname{Re}}\left(\frac{\partial^{2} V_{z}}{\partial r^{2}}+\frac{1}{r} \frac{\partial V_{z}}{\partial r}+\frac{\partial^{2} V_{z}}{\partial z^{2}}\right), \\
\frac{\partial V_{\varphi}}{\partial t}+V_{z} \frac{\partial V_{\varphi}}{\partial z}+V_{r} \frac{\partial V_{\varphi}}{\partial r}+\frac{V_{r} V_{\varphi}}{r}=\frac{1}{\operatorname{Re}}\left(\frac{\partial^{2} V_{\varphi}}{\partial r^{2}}+\frac{1}{r} \frac{\partial V_{\varphi}}{\partial r}+\frac{\partial^{2} V_{\varphi}}{\partial z^{2}}-\frac{V_{\varphi}}{r^{2}}\right),
\end{gathered}
$$




$$
\begin{gathered}
\frac{\partial V_{r}}{\partial t}+V_{z} \frac{\partial V_{r}}{\partial z}+V_{r} \frac{\partial V_{r}}{\partial r}-\frac{V_{\varphi}^{2}}{r}=-\frac{\partial p}{\partial r}+\frac{1}{\operatorname{Re}}\left(\frac{\partial^{2} V_{r}}{\partial r^{2}}+\frac{1}{r} \frac{\partial V_{r}}{\partial r}+\frac{\partial^{2} V_{r}}{\partial z^{2}}-\frac{V_{r}}{r^{2}}\right), \\
\frac{1}{r} \frac{\partial\left(V_{r} r\right)}{\partial r}+\frac{\partial V_{z}}{\partial z}=0
\end{gathered}
$$

where $V_{z}, V_{r}, V_{\varphi}$ are the axial, radial and azimuthal velocity components, $p$ is the pressure, $\operatorname{Re}$ is the Reynolds number.

Suppose that the flow under consideration has the following velocity distribution:

$$
V_{z}=U(r), \quad V_{r}=0, \quad V_{\varphi}=W(r)
$$

We will investigate the behavior of small perturbations for flow (5) in the form:

$$
\left\{V_{z}^{\prime}, V_{r}^{\prime}, V_{\varphi}^{\prime}, p^{\prime}\right\}=\{F, i S, H, P\} \exp [i(\alpha z+n \varphi-\alpha c t)],
$$

where $\alpha$ is the wave number; $n$ is the disturbance mode $(n=0 ; \pm 1 ; \pm 2 ; \ldots), c$ is the wave propagation velocity; $i$ is the imaginary unit; $F(r), S(r), H(r), P(r)$ are the complex amplitude functions.

After linearization equations (1)-(4) and substitution expressions (5), (6), we obtain the ordinary differential equations system for the complex amplitude functions:

$$
\begin{gathered}
r^{2} \gamma F+\alpha r^{2} P+r^{2} S U^{\prime}=\frac{1}{i \operatorname{Re}}\left[r\left(r F^{\prime}\right)^{\prime}-\left(\alpha^{2} r^{2}+n^{2}\right) F\right], \\
r^{2} \gamma S+2 r H W-r^{2} P^{\prime}=\frac{1}{i \operatorname{Re}}\left[r\left(r S^{\prime}\right)^{\prime}-\left(\alpha^{2} r^{2}+n^{2}+1\right) S-2 n H\right], \\
r^{2} \gamma H+r^{2} S\left(W^{\prime}+\frac{W}{r}\right)+r n P=\frac{1}{i \operatorname{Re}}\left[r\left(r H^{\prime}\right)-\left(\alpha^{2} r^{2}+n^{2}+1\right) H-2 n S\right], \\
\alpha r F+(r S)^{\prime}+n H=0,
\end{gathered}
$$

where $\gamma=\alpha(U-c)+n W / r$.

The boundary conditions at $r=0$ for system (7)-(10) can be written in the form:

$$
\begin{gathered}
S(0)=H(0)=0, F(0), P(0)-\text { bounded for } n=0, \\
S(0) \pm H(0)=0, \quad F(0)=P(0)=0-\text { for } n= \pm 1, \\
S(0)=H(0)=F(0)=P(0)=0 \quad-\text { for }|n|>1 .
\end{gathered}
$$

The conditions on the pipe wall at $r=1$ have the form:

$$
F(1)=S(1)=H(1)=0 \text {. }
$$

For free vortex flows (unbounded by solid walls), the damping conditions for disturbances are applied 


$$
S(\infty)=H(\infty)=F(\infty)=P(\infty)=0
$$

In this study, perturbations (6) are considered periodic in $z$ with a time-varying amplitude. So $\alpha$ is a real value ( $\alpha=2 \pi / \lambda$, where $\lambda$ is the perturbation wavelength), and $c=c_{r}+i c_{i}$ is a complex value, $c_{r}$ is the propagation disturbance velocity in $z$ direction (phase speed), $c_{i}$ is the growth rate disturbance in time. The amplitudes of the disturbance (6) decay (the flow is stable), if $c_{i}<0$, and grow with time (the flow is unstable), if $c_{i}>0$.

\section{Numerical method}

We represent system (7)-(10) in matrix form:

$$
\begin{aligned}
& \frac{d z_{i}}{d r}=A_{i, j} z_{j}, \quad z_{j}=\left\{F, S, H, r P, r F^{\prime}, r H^{\prime}\right\},
\end{aligned}
$$

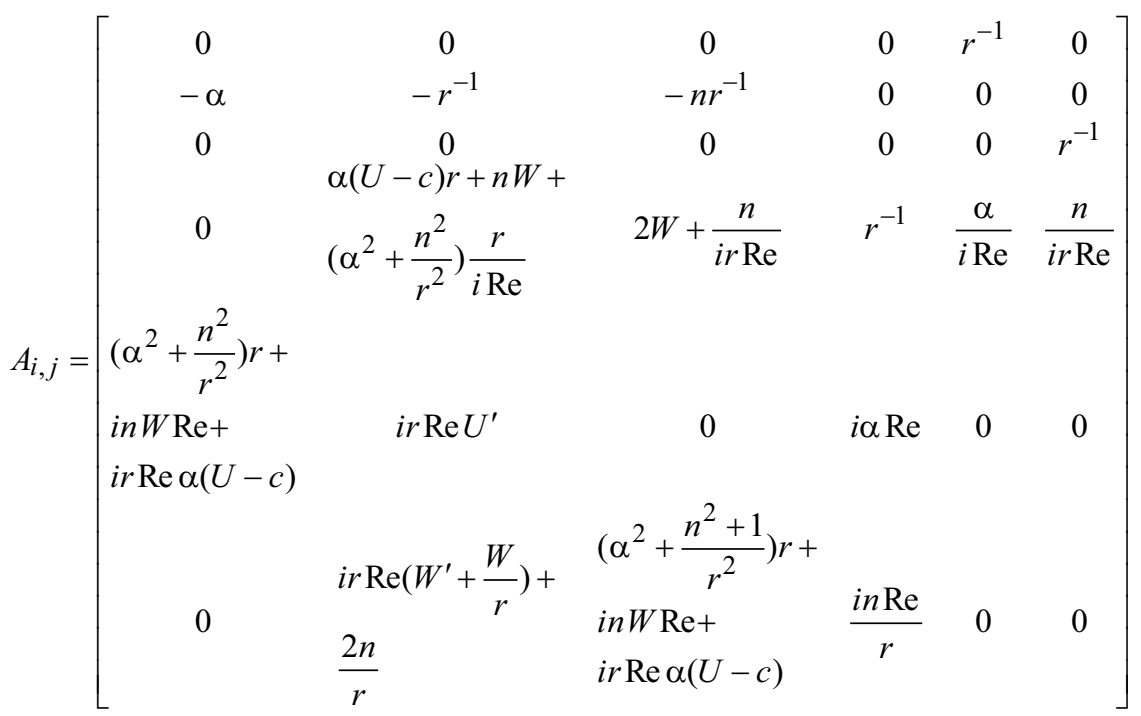

The resulting system (16) has a regular singular point at $r=0$. In the general case, a linear system of differential equations has a regular singular point if we can write the original system in the form:

$$
r y_{p}^{\prime}(r)=\sum_{q=1}^{n} f_{p, q}(r) y_{q}, \quad p=1,2, \ldots n
$$

where $f_{p, q}(r)=\sum_{v=0}^{\infty} a_{p, q}^{(v)} r^{\nu}$ and all functions at the point $r=0$ are regular and do not vanish simultaneously. The solution can be found by the Frobenius method in the power series form:

$$
y_{1}=r^{\lambda} \varphi_{1}(r), \ldots, y_{n}=r^{\lambda} \varphi_{n}(r), \quad \varphi_{p}(r)=\sum_{v=0}^{\infty} c_{p, v} r^{\nu}
$$


where $\lambda$ is the root of the characteristic equation

$$
\operatorname{det}\left(f_{p, q}-\lambda e_{p, q}\right)=0, \quad e_{p, q}= \begin{cases}1, & p=q \\ 0, & p \neq q\end{cases}
$$

Let's use the Frobenius method to find the solution in this case. We write system (16) in the form:

$$
r z^{\prime}=\left(\sum_{k=0}^{\infty} A_{k} r^{k}\right) z
$$

where matrices $A_{k}$ consist of coefficients at powers $r^{k}$. We will find the solution in the form:

$$
z=r^{\lambda} \sum_{k=0}^{\infty} s_{k} r^{k}
$$

Substituting it into the system of equations (16), we obtain:

$$
\sum_{k=0}^{\infty}(\lambda+k) s_{k} r^{k}=\sum_{k=0}^{\infty} A_{k} r^{k} \cdot \sum_{k=0}^{\infty} s_{k} r^{k}
$$

Equating the coefficients at the same degrees, we obtain the following matrix system for vectors:

$$
\begin{aligned}
& \left(A_{0}-\lambda E\right) s_{0}=0, \\
& \left(A_{0}-(\lambda+1) E\right) s_{1}=-A_{1} s_{0}, \\
& \left(A_{0}-(\lambda+2) E\right) s_{2}=-A_{2} s_{0}-A_{1} s_{1}, \\
& \cdots \cdots \quad \text { etc }
\end{aligned}
$$

where $E$ is the identity matrix. The characteristic equation allows you to find $\lambda$ : $\pm(n+1), \pm n, \pm(n-1)$. Three of them should be discarded, since the perturbations should be bounded at $r=0$. We will number the remaining three values in such a way that the condition $\lambda_{1}>\lambda_{2}>\lambda_{3}$ is satisfied. After finding the expansion coefficients, we get the fundamental solutions system. To determine the coefficients, a computer program was developed that allows to calculate any number of terms in the expansion (18). Finding the coefficients is reduced to solving systems of linear equations.

The stability problem for swirling flows unbounded by solid walls has one more singular point at $r \rightarrow \infty$. To find a solution in this case, we write the system of equations (7)-(10) in the form:

$$
\frac{d y_{i}}{d r}=\hat{A}_{i, j} y_{j}, \quad y_{j}=\left\{F, S, H, P, F^{\prime}, H^{\prime}\right\}
$$

In most cases, at large values of $r$ for a free vortex we can take $U=0, W=q / r$. Then the matrix $\hat{A}_{i, j}$ will have the form: 


$$
A_{i, j}=\left[\begin{array}{cccccc}
0 & 0 & 0 & 0 & 1 & 0 \\
-\alpha & -r^{-1} & -n r^{-1} & 0 & 0 & 0 \\
0 & 0 & 0 & 0 & 0 & 1 \\
0 & -\frac{i}{\operatorname{Re}}\left(\beta^{2}+\frac{n^{2}}{r^{2}}\right)+\frac{n q}{r^{2}} & 2 \frac{q}{r^{2}}-\frac{i n}{r^{2} \operatorname{Re}} & 0 & -\frac{i \alpha}{\operatorname{Re}} & -\frac{i n}{r \operatorname{Re}} \\
\frac{n q i \operatorname{Re}}{\beta^{2}} & 0 & 0 & i \alpha \operatorname{Re} & -\frac{1}{r} & 0 \\
0 & \frac{2 n}{r^{2}} & \beta^{2}+\frac{n^{2}+1}{r^{2}}+ & \frac{i n \operatorname{Re}}{r} & 0 & -\frac{1}{r}
\end{array}\right]
$$

where $\beta^{2}=\alpha^{2}-i \alpha c \operatorname{Re}$. The solution at this irregular singular point will be sought in the form

$$
y_{i}=e^{\lambda r} r^{D} \sum_{v=0}^{\infty} b_{i, v} r^{-v}
$$

To determine the expansion coefficients, it is necessary to solve the system of matrix equations:

$$
\begin{aligned}
& \left(B_{0}-\lambda E\right) b_{0}=0 \\
& \left(B_{0}-\lambda E\right) b_{1}=-\left(B_{1}-D E\right) b_{0}, \\
& \left(B_{0}-\lambda E\right) b_{2}=-\left(B_{1}-(D-1) E\right) b_{1}-B_{2} b_{0}, \\
& \left(B_{0}-\lambda E\right) b_{3}=-\left(B_{1}-(D-2) E\right) b_{2}-B_{2} b_{1}-B_{3} b_{2}, \\
& \cdots \cdots \text { etc }
\end{aligned}
$$

Here $B_{v}$ is the matrix consisting of the expansion coefficients of the matrix $\hat{A}$ in powers $r^{-v}$, i.e. $\hat{A}=\sum_{v=0}^{\infty} B_{v} r^{-v}, b_{v}$ is the vector of the required coefficients. From the first equation (21) follows the equation for determining $\lambda$ :

$$
\operatorname{det}\left(B_{0}-\lambda E\right)=0
$$

Therefore we find 6 values $\lambda: \pm \alpha, \pm \beta, \pm \beta$. Three of them $\lambda=\alpha,+\beta,+\beta$ should be discarded, since the perturbations should vanish at $r=\infty$. For all of the three $\lambda, D=0.5$ is obtained.

In the particular case, for $\lambda=-\alpha$, the solution can be written in the form of modified Bessel functions

$$
\{F, S, H, P\}=\left\{K_{n}(\alpha r),-\frac{K_{n}^{\prime}(\alpha r)}{\alpha}, \frac{n K_{n}(\alpha r)}{\alpha r},\left(c-\frac{n q}{\alpha r^{2}}\right) K_{n}(\alpha r)\right\},
$$


where

$$
\begin{aligned}
K_{n}(\alpha r)= & e^{-\alpha r} r^{-1 / 2}\left\{1+\frac{\left(n^{2}-1 / 4\right)}{1 ! \cdot 2} \frac{1}{\alpha r}+\frac{\left(n^{2}-1 / 4\right)\left(n^{2}-9 / 4\right)}{2 ! \cdot 2^{2}} \frac{1}{(\alpha r)^{2}}+\right. \\
& \left.+\frac{\left(n^{2}-1 / 4\right)\left(n^{2}-9 / 4\right)\left(n^{2}-25 / 4\right)}{3 ! \cdot 2^{3}} \frac{1}{(\alpha r)^{3}}+\ldots\right\} .
\end{aligned}
$$

\section{Calculation results and discussion}

The considered asymptotic method allows us to find a solution for the system of equations (7)-(10) in the neighborhood of singular points. Using these expansions, the integration of equations (7)-(10) was performed by the Runge-Kutta method with an automatic choice of the step. To ensure the stability of calculations, the Gram-Schmidt orthogonalization method was applied. The software package implementing this algorithm was compiled in the Fortran-90 programming language.

As a test problem, we investigate flow stability in a tube rotating around its axis at constant angular velocity $q$ :

$$
U(r)=1-r^{2}, \quad W(r)=q r .
$$

The calculated dispersion curves for a fixed Reynolds number $\operatorname{Re}=1000$ and $q=1$ are shown in Figure 1. In this case, there are five unstable modes. All these modes are inviscid, since the amplification coefficients tend to some constant values at $\operatorname{Re} \rightarrow \infty$. The calculated values $\omega_{i}=\alpha c_{i}$ and $\omega_{r}=\alpha c_{r}$ at large values of the Reynolds number $\operatorname{Re}=10^{5}$ correspond to the results previously obtained using the inviscid theory.

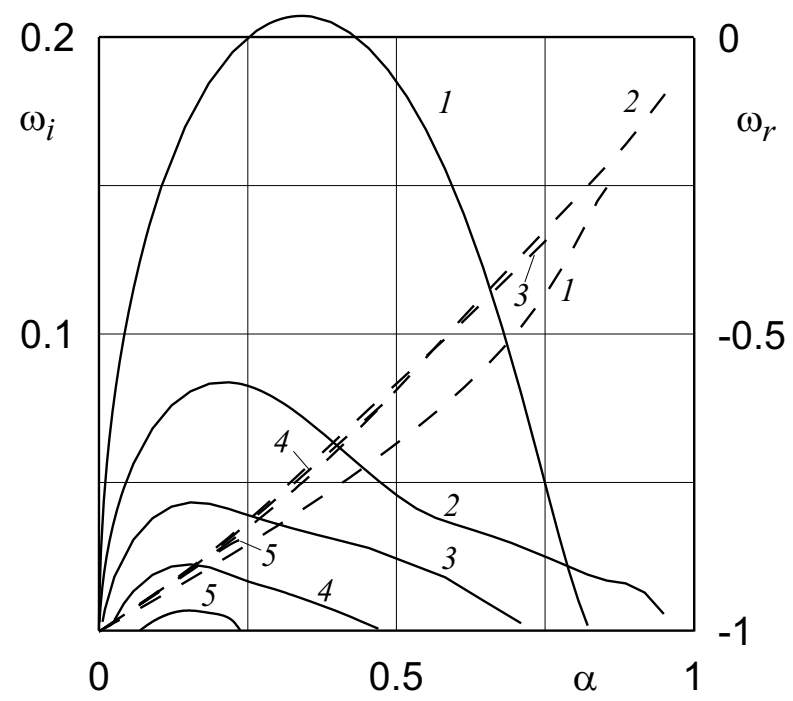

Fig. 1. Amplification coefficients (solid lines) and oscillation frequency (dashed lines) at $\operatorname{Re}=10^{4}$, $q=1$, modes $1-5$ (curves $1-5$ ) 


\section{Conclusions}

The developed algorithm makes it possible to investigate the stability of swirling flows with an arbitrary velocity profile and to determine the range of the fastest growing disturbances. Analysis of the calculated dependences contributes to the correct choice of the optimal operating mode of vortex devices in order to avoid the phenomenon of vortex destruction.

The work was supported financially by the Russian Foundation for Basic Research (project No 18-0100762).

\section{References}

1. A. Gupta, G. Lilley, N. Syred, Swirl Flows (Abacus Press, 1984)

2. S.V. Alekseenko, P.A. Kuibin, V.L. Okulov, Introduction to theory of concentrated vortices (Institute of Computer Science, Moscow, 2005)

3. N. Syred, Prog. Energy. Combust. Sci., 32, 93 (2006)

4. P. Moise, J. Mathew, Fluid Mech., 873, 322 (2019)

5. Z. Mansouri, M. Aouissi, T.Boushaki, Int. J. Heat Technol., 34 (2), 227 (2016)

6. V.Ya. Karelin, G.I. Krivchenko, A.P. Mordasov, V.V. Volshanik, A.L. Zuikov, V.K. Akhmetov, Physical and mathematical modeling of energy extinguishing systems in vortex spillways, in Proceedings of the conference MG-89, 24-26 May 1989, Divnogorsk, Russia (1989)

7. V.K. Akhmetov, V.V. Volshanik, Hydr. Construction, 28 (10), 605 (1994)

8. J. M. Lopez, Phys. Fluids, 24, 014101 (2012)

9. M. Bauerheim, F. Nicoud, T. Poinsot, Phys. Fluids, 28, 021303 (2016)

10. G. Balestra, M. Gloor, L. Kleiser, Phys. Fluids, 27, 054101 (2015)

11. V.K. Akhmetov, V.Ya. Shkadov, Mosc. Univ. Mech. Bull., Ser. I, 58 (1), 23 (2003)

12. M.A. Herrada, V.N. Shtern, J.M. López-Herrera, 25, 093604 (2013)

13. I. Delbende, M. Rossi, Phys. Fluids, 17, 044103 (2005)

14. V.K. Akhmetov, V.Ya. Shkadov, Mosc. Univ. Mech. Bull., Ser. I, 42 (2), 17 (1987)

15. F. Gallaire, J.-M. Chomaz, Phys. Fluids, 15, 2622 (2003)

16. F.J. Blanco-Rodríguez, J.O. Rodríguez-García, L. Parras, C. del Pino, Phys. Fluids, 29, 064108 (2017)

17. N.K. Yadav, A. Samanta, Fluid Mech., 823, 689 (2017)

18. I. Delbende, M. Rossi, Phys. Fluids, 17, 044103 (2005) 